\title{
Bonnes résolutions de Nouvel An: que sont-elles devenues?
}

\section{Bruno Kesseli}

Dr méd. et lic. phil., rédacteur en chef

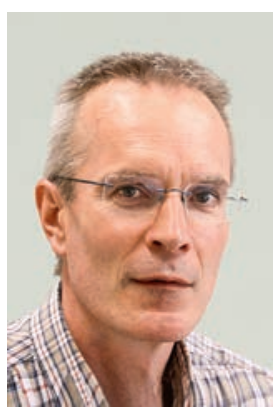

Avez-vous, il y a environ un mois, commencé la nouvelle année en prenant de bonnes résolutions? Dans l'affirmative, le moment n'est peut-être pas mal choisi pour dresser un petit bilan intermédiaire. Si celui-ci s'avère décevant, vous êtes sans doute en bonne compagnie. Je ne tire pas cette conclusion d'une étude, elle est simplement basée sur la réalité quotidienne. En ce qui me concerne, je ne me souviens pas avoir jamais pris une bonne résolution à Nouvel An. Je ne peux pas vraiment dire à quoi cela tient. Une théorie plausible serait que je les oublie immédiatement, dans l'agitationéventuellement légèrement alcoolisée - de la fête.

En tant que médecin aussi, on est régulièrement confronté aux bonnes résolutions de santé de ses patients.

Quoi qu'il en soit, cela ne signifie pas que je ne prends pas de résolutions dans la vie. Et mon taux de réussite n'est probablement pas plus élevé que celui des gens qui les associent à la nouvelle année. Mais mes résolutions me sont généralement inspirées par une lecture ou une discussion passionnante. Je tiens alors à les mettre immédiatement en pratique, sans attendre le $1^{\text {er }}$ janvier. Cette attente me semblerait d'autant moins judicieuse que beaucoup de ces résolutions ont pour but d'améliorer la santé, donc la joie de vivre.

En tant que médecin aussi, on est régulièrement confronté aux bonnes résolutions de santé de ses patients. Dans ce contexte, il serait bon de savoir s'il existe des astuces basées sur les faits pour augmenter les probabilités de mise en pratique durable des bonnes résolutions. Ces stratégies pourraient alors être communiquées aux patients.

Je suis récemment tombé sur un article intéressant à ce sujet de la série "Reality Check» [1] de la BBC. Il résume les conclusions de divers chercheurs en sciences sociales, sciences humaines et neurosciences, dont certaines sont surprenantes, du moins à mes yeux. Ainsi, les chances de réussir semblent meilleures lorsqu'il s'agit de retrouver quelque chose de "perdu» «perdu» peut être un loisir abandonné ou un niveau de forme antérieur, par exemple. L'article postule que le mécanisme en jeu ici est une "aversion de la perte» inhérente à l'être humain. Conclusion moins étonnante: les résolutions doivent être réalistes, et il est préférable de les préciser en détail. «Manger du poisson tous les vendredis» est plus pertinent que simplement «manger plus de poisson». Les exceptions devraient être fixées dès le départ pour qu'il soit moins facile d'inventer des excuses ad hoc. La promenade est par exemple facultative le jour de son anniversaire, mais pas quand il pleut. Les résolutions les plus couronnées de succès sont apparemment celles qui rendent service à un proche ou simplement qui impliquent d'autres personnes. Avoir sa réputation ternie en cas de non-respect de la résolution est également un facteur de motivation. Rendre dans une certaine mesure ses résolutions publiques peut donc être une bonne idée. Bref, s'en remettre à sa volonté suffit rarement, la planification fait (presque) tout.

\section{L'article intitulé «Do health professionals keep} their new year's resolutions?» devrait apporter des réponses.

«PubMed» ne liste pas moins de 70 articles avec les mots-clés «New Year's resolutions». Je vous aurais volontiers fait part du taux de réussite chez les médecins. L’article intitulé «Do health professionals keep their new year's resolutions?», librement accessible en plein texte, devrait apporter des réponses. Mais après lecture de la première phrase j'ai malheureusement dû interrompre l'exercice. J'espère que vous ne m'en voudrez pas. Il débute en effet ainsi: Livsstilssjukdomar står för en hög andel av sjuklighet $i$ västvärlden, och när WHO rankade de tio ledande dödsorsakerna i världen 2014 hade alla listade dödsorsaker - åtminstone delvis - livsstilsrelaterade riskfaktorer [2].

1 New Year's resolutions: How do you make one you will keep? www.bbc.com/news/uk-42353226

2 Ludvigsson JF. Håller vårdpersonal sina nyårslöften? [Do health professionals keep their new year's resolutions?]. Läkartidningen. 15 déc. 2015;112. http://lakartidningen.se/Aktuellt/Kultur/ Kultur/2015/12/Haller-vardpersonal-sina-nyarsloften/ 